\title{
Reanálisis del estudio 329: ni eficacia ni seguridad de la paroxetina e imipramina para la depresión mayor en adolescentes
}

Reanalysis of the study 329: neither efficacy nor safety of paroxetine and imipramine for major depression in adolescents

Le Noury J, y col. BMJ 2015;351:h4320.

\section{Objetivos}

Reanalizar el estudio 329 publicado por Kellery col.' ${ }^{1}$ ayyo objetivo primanio fue evatuar la eficacia y segunidad del tratamiento de la depresión mayor unipolar en adolescentes con paroxetinaeimipraminaen comparación con placebo. Elestudio329 condiuyó quelaparoxetinaes seguray eficaz Egnupo de restauración de ensayos dínicos invisibles y abandonados (en ingles RIAT) identifico sesgos en la publicación, por lo que se propuso reanalizar los datos.

\section{Diseño y lugar}

Se reanalizaron los datos del estudio 329 siguiendo estrictamente su protocolo y las recomendaciones RIAT. El estudio 329 fue un ensayo clínico controlado aleatorizado, doble ciego, multicéntrico realizado entre los años 1994 y 1998 en doce centros psiquiátricos de EE.UU. y Canadá.

\section{Participantes}

Incluyó 275 adolescentes de 12 a 18 años con diagnóstico de depresión mayor, según criterios del DSM-III-R (similar al IV), de al menos ocho semanas de duración.

\section{Intervención}

Se aleatorizaron a los participantes a recibir diariamente paroxetina (20

Tabla 1: Resultados primarios de eficacia.

\begin{tabular}{l|c|c|c|c}
\multicolumn{1}{c|}{ Variables } & Paroxetina (n=93) & Imipramina (n=95) & Placebo (n=87) & p \\
\hline Cambio de puntaje HAM-D (IC95\%) & $-10,7(-12,3 \mathrm{a}-9,1)$ & $-9(-10,7 \mathrm{a}$ 7,5) & $-9,1(-10,7 \mathrm{a} 7,5)$ & 0,20 \\
\hline Proporción de respondedores & $66,7 \%(60 / 30)$ & $58,5 \%(55 / 39)$ & $55,2 \%(48 / 39)$ & 0,27 \\
\hline
\end{tabular}

Tabla 2: Numero de eventos adversos reportados.

\begin{tabular}{|c|c|c|c|c|c|c|c|c|c|}
\hline \multirow[b]{2}{*}{ Eventos adversos } & \multicolumn{3}{|c|}{ Paroxetina $(n=93)$} & \multicolumn{3}{|c|}{ Imipramina $(n=95)$} & \multicolumn{3}{|c|}{ Placebo $(n=87)$} \\
\hline & CSR $^{*}$ & Keller* & RIAT\# & CSR $^{*}$ & Keller* & RIAT\# & CSR $^{*}$ & Keller* & RIAT\# \\
\hline Cardiovasculares & 7 & 5 & 44 & 60 & 42 & 130 & 12 & 6 & 32 \\
\hline Psiquiátricos & - & - & 103 & - & - & 63 & - & - & 24 \\
\hline Totales & 338 & 265 & 481 & 493 & 340 & 552 & 277 & 207 & 330 \\
\hline
\end{tabular}

CSR: reporte del ensayo clínico. *Codificados con ADECS (adverse drug events coding system) o \#MedDRA

\section{Conclusiones}

La revisión del estudio 329 no mostró ninguna ventaja de la paroxetina o la imipramina sobre el placebo en téminos de eficacia para el tratamiento de la a $40 \mathrm{mg}$ ), imipramina (200 a $300 \mathrm{mg}$ ) o placebo, durante ocho semanas.

\begin{abstract}
Medición de resultados principales
Los resultados primarios de eficacia fueron el cambio de la puntuación de la escala de depresión de Hamilton (HAM-D) desde el inicio hasta el final del tratamiento (de al menos cuatro puntos o más) y la proporción de respondedores (HAM -D $\leq 8$ o reducción $\geq 50 \%$ del HAM-D basal) al final del estudio. Para evaluar seguridad, se codificaron los téminos literales descriptos en el reporte del ensayo clínico (CSR) 329 y lo originalmente publicado, mediante el sistema de codificación ADECS y además con un minucioso diccionario médico llamado MedDRA, evaluándose los formularios de reporte de caso de 93 (34\%) pacientes. Se valoró la diferencia en el número de eventos descritos con los tres métodos.
\end{abstract}

\section{Resultados principales}

La eficacia de la paroxetina y la imipramina no fue estadística ni clínicamente diferente en comparación con placebo (tabla 1). Se detectaron mayor cantidad de efectos adversos y efectos adversos graves que no habían sido reportados en el artículo publicado por Keller y col1, independientemente del método de análisis (tabla 2). Los mismos incluyeron ideación y comportamiento suicida en la rama paroxetina y problemas cardiovasculares en el grupo de la imipramina. depresión en adolescentes y se detectaron mayor cantidad de efectos adversos y efectos adversos graves en ambas ramas del estudio.

Fuente de Financiamiento: No refiere.

\section{Comentario}

El $20 \%$ de los adolescentes de todo el mundo tienen problemas psiquiátricos, la depresión es la que más contribuye a la carga mundial de morbilidad y el suicidio es una de las tres causas principales de mortalidad entre esta población ${ }^{3}$. Tener información científica clara sobre su tratamiento es de vital importancia para su correcto abordaje. El estudio 329 fue un estudio influyente en la práctica médica y permitió posicionar a la paroxetina como el antidepresivo más vendido en EE.UU. en el año $2001^{4}$. La iniciativa internacional RIAT ${ }^{5}$ propone reanálisis independientes de ensayos clínicos con sesgos potenciales en el diseño o los resultados. En el reanálisis del estudio 329 se ponen en evidencia diferentes sesgos. Principalmente se evidencian sesgos de reporte de la efectividad y daños. Con respecto a la efectividad, el estudio 329 concluye que la paroxetina es efectiva para el tratamiento de la depresión en adolescentes, sustentado en cuatro variables no especificadas en el protocolo e informadas con diferencias estadísticamente significativas.

Laura Fraguas [ Servicio de Medicina Familiar del Hospital Italiano de Buenos Aires. laura.fraguas@ hospitalitaliano.org.ar]

Fraguas L. Reanálisis del estudio 329: ni eficacia ni seguridad de la paroxetina e imipramina para la depresión mayor en adolescentes. Evid Act Pract Ambul. $2016 ; 19(3): 76$. Comentado de: Le Noury J, y col. Restoring Study 329: efficacy and harms of paroxetine and imipramine in treatment of major depression in adolescence. BMJ. 2015;351:h4320. PMID: 26376805.

Referencias:

1. Keller MB, Ryan ND, Strober M, et al. Efficacy of paroxetine in the treatment of adolescent major depression: a randomized, controlled trial. J Am Acad Child Adolesc Psychiatry2001;40:762-72. 2. Diagnostic and statistical manual of mental disorders, third edition, revised (DSM-III-R). American Psychiatric Association, 1987.

3. UNICEF, La adolescencia una época de oportunidades, EEUU, 2011.

4. http://study329.org/

5. Doshi P, Dickersin K, Healy D, Vedula SS, Jefferson T. Restoring invisible and abandoned trials: a call for people to publish the findings. BMJ2013;346:f2865. 